\title{
Utilização do ensino à distância como ferramenta de capacitação e formação para técnico em saúde bucal
}

\author{
Maria Isabel de Castro de Souza*, Marcia Rendeiro**, Renata Rocha Jorge***, KatlinDarlen \\ Maia****, Aline Borges Luiz Monnerat*****, Maria Rita dos Santos Ritto******. \\ * Professora Associada do Departamento de Odontologia Preventiva e Comunitá- \\ ria/FOUERJ \\ Coordenadora do Curso de TSB/FOUERJ \\ ** Professora Adjunta do Departamento de Odontologia Preventiva e Comunitá- \\ ria/FOUERJ \\ *** Professora Adjunta do Departamento de Odontologia Preventiva e Comunitá- \\ ria/FOUERJ \\ **** Professora Adjunta do Departamento de Odontologia Preventiva e Comunitá- \\ ria/FOUERJ \\ ***** Coordenadora Adjunta do Curso de TSB/ FOUERJ \\ ****** TSB - Supervisora técnica do Curso de TSB/FOUERJ
}

\section{RESUMO}

O objetivo deste presente estudo foi analisar e apresentar, por meio de um relato de caso, a aplicabilidade do Ensino à Distância como ferramenta de trabalho na formação e capacitação de profisssionais de nível técnico (Técnico em Saúde Bucal) da área de Odontologia.A proposta de construção do modelo de cursofoi baseada na necessidade de formação em larga escala, visando atender à implementação rápida $\mathrm{e}$ progressiva da Política Nacional de Saúde Bucal. Foram realizadas duas turmas piloto, para verificar a adesão e aplicabilidade do modelo à distância, para formar novos profissionais, bem como para atender aqueles que já se encontram inseridos no mercado de trabalho. Após a realização das duas turmas, $\quad 100 \%$ dos alunos inscritos concluíram o curso. Na turma 1, $100 \%$ recomendariam o curso e da turma 2 , $85 \%$ o fariam. Sobre a realização de concurso público, contabilizando as duas turmas, $80 \%$ foram aprovados. Diante dos resultados, consideramos que a educação a distân- cia é uma ferramenta potente para atender as demandas para a formação profissional técnica em Odontologia, na perspectiva do SUS e da legislação setorial.

Descritores: Ensino à distância, SUS, Saúde Bucal Coletiva, Tecnologia de Informação, Técnico em Saúde Bucal

\section{INTRODUÇÃO}

Com a globalização, onde a informática assume cada vez maior importância na redefinição de relações de produção e nas relações sociais como um todo, são inevitáveis e fundamentais as transformações no campo educacional, tornando essencial utilizar um enfoque pedagógico voltado para a Tecnologia Educacional que inclua iniciativas de Educação a Distância $(\mathrm{EAD})^{2}$. E essa abordagem deve ser orientada por uma liderança acadêmica que operacionalize sua visão nesta direção, definindo suas metas e objetivos que devem ser trabalhados pedagogicamente mediante a concretização de 
uma política de organização pedagógica e acadêmica adequada. ${ }^{10}$

Segundo pesquisa recente ${ }^{17}$ o acesso dos profissionais às ferramentas eletrônicas para informação tem se mostrado satisfatório, sendo necessário promover o estímulo para um grupo ainda pouco habituado a esta importante ferramenta de educação permanente.

O uso de tecnologias de informação e comunicação, bem como de metodologias não tradicionais na educação, já não é mais novidade nem mesmo para pequenas instituições educativas. ${ }^{14}$ Entretanto, o desenvolvimento de um Sistema Educacional que conjugue estes instrumentos e ideias, com base em um rigoroso conceito de qualidade e na necessária dialogicidade, que seja capaz de incentivar o desenvolvimento de redes colaborativas, integrando sujeitos e extrapolando os conceitos de espaço-tempo, que vise à superação de modelos tradicionais da Educação a Distância, este sim, é um grande desafio. ${ }^{6}$ Neste sentido recorremos a Perez $^{13}$ (2002) que nos lembrou que os ambientes virtuais de aprendizagem, nos quais são desenvolvidos os cursos online, podem ser integrados a cursos totalmente a distância, semipresenciais e presenciais. Porém, qualquer que seja a modalidade, requer uma redefinição dos elementos organizativos do processo de aprendizagem em relação aos agentes envolvidos (docentes, alunos, pessoal administrativo), e aos espaços onde acontece o processo educativo, nos tempos e sequências de aprendizagem.

Nesta abordagem online, os métodos e planos pedagógicos situam o aluno no centro do processo de aprendizagem como um aprendiz que define de maneira autônoma seu caminho de aprendizagem, construindo seu conhecimento por meio da interação com os materiais, com a tecnologia, com o tutor e com os companheiros. Isso ocorre devido ao fato de o acesso cada vez maior às tecnologias e, consequentemente, às informações e facilidades de comunicação possibilitar que o estudante seja capaz de definir seu próprio percurso e de, efetivamente, tomar parte no processo de construção do conhecimento, permitindo ao professor o desempenho de um papel mais dinâmico e menos dogmático ${ }^{15}$.

Em outras palavras, a viabilidade para os educadores contemporâneos de converter o tradicional sistema deensino, onde o professor expõe e o aluno "absorve" suas ideias, em outro onde "todos estudam" e constroem o conhecimento cooperativamente pode ser a grande transformação da educação para o futuro.

Podemos descrever algumas características do ensino a distância, em relação ao curso de graduação:

- Interatividade: em salas de aula, a interatividade fica restrita ao limite físico e temporal, o que não ocorre com os recursos pela Web, em que não há restrições de localização e, quanto ao fator temporal, o estudante pode acessar a Internet a qualquer horário.

- Ensino independente de tempo e lugar: o treinamento pode ser feito em qualquer lugar a qualquer momento, como explicado no item anterior. $\mathrm{O}$ alcance é limitado pelo alcance da Internet.

- Minimização de deslocamentos: não há necessidades de deslocamentos frequentes para locais físicos predeterminados, o que também determina uma redução de custos sobre o transporte, alimentação e hospedagem.

- Economia de tempo: por haver uma minimização de deslocamentos, há, consequentemente, uma redução de tempo.

- Atendimento em massa personalizado 
(masscustomization): é a possibilidade de oferecimento de ensino em massa com a adequação às características dos alunos.

- Possibilidade de determinação, pelo aluno, de seu ritmo de aprendizagem: o que faz com que o indivíduo possa ter um papel ativo sobre o ritmo e necessidades de aprendizagem.

- Network: possibilita e promove a troca de experiências, criando uma comunidade virtual. Essa troca enriquece, estimula e dinamiza o aprendizado dos alunos. ${ }^{6}$

Historicamente, a evolução da prática odontológica conduziu ao aumento das atribuições do Cirurgião-Dentista no consultório, tornando-se portanto, necessária a presença de outros profissionais auxiliares, o que assegura o mínimo de conforto e a qualidade do tratamento de seus pacientes ${ }^{12}$.

Conforme onúmero de pacientes aumenta, o dentista necessita de profissionais competentes que possam auxiliar na organização do consultório, fazendo trabalhos indiretos, permitindo assim que o mesmo se concentre nos pacientes bem como nos planejamentos dos $\operatorname{casos}^{12}$.

No Brasil, em 1975, o atendente de consultório dentário (hoje Auxiliar em Saúde Bucal) e técnico em higiene dental (hoje Técnico em Saúde Bucal) tiveram sua nomenclatura e papel definitivamente estabelecidos, com a definição formal dos seus perfis e currículos mínimos pelo Ministério da Educação (Parecer no 460/75 para atendente de consultório dentário e técnico em higiene dental $)^{3}$. O parecer do Ministério da Educação e Cultura (MEC) e Conselho Federal de Educação estabelece a habilitação do atendente de consultório dentário e técnico em higiene dental e descreve suas ocupações, os requisitos essenciais para o exercício de sua função e os currículos do seu curso de formação ${ }^{3}$.
Após anos de luta e devido à tamanha importância de tais profissionais, eles foram legalizados no país somente no ano de 2008 , através da lei $\mathrm{n}^{\circ} 11.889 / 2008$ do governo federal brasileiro ${ }^{4}$.

De acordo com a lei ${ }^{4} 11.889 / 2008$, competem ao Técnico em Saúde Bucal, sempre sob a supervisão do cirurgiãodentista, as seguintes atividades, além das estabelecidas para os auxiliares em saúde bucal:

I - participar do treinamento e capacitação de Auxiliar em Saúde Bucal e de agentes multiplicadores das ações de promoção à saúde;

II - participar das ações educativas atuando na promoção da saúde e na prevenção das doenças bucais;

III - participar na realização de levantamentos e estudos epidemiológicos, exceto na categoria de examinador;

IV - ensinar técnicas de higiene bucal e realizar a prevenção das doenças bucais por meio da aplicação tópica do flúor, conforme orientação do cirurgião-dentista;

V - fazer a remoção do biofilme, de acordo com a indicação técnica definida pelo cirurgião-dentista;

VI - supervisionar, sob delegação do cirurgião-dentista, o trabalho dos auxiliares de saúde bucal;

VII - realizar fotografias e tomadas de uso odontológicos exclusivamente em consultórios ou clínicas odontológicas;

VIII - inserir e distribuir no preparo cavitário materiais odontológicos na restauração dentária direta, vedado o uso de materiais e instrumentos não indicados pelo cirurgião-dentista;

IX - proceder à limpeza e à antissepsia do campo operatório, antes e após atos cirúrgicos, inclusive em ambientes hospitalares;

$\mathrm{X}$ - remover suturas; 
XI - aplicar medidas de biossegurança no armazenamento, manuseio e descarte de produtos e resíduos odontológicos;

XII - realizar isolamento do campo operatório;

XIII - exercer todas as competências no âmbito hospitalar, bem como instrumentar o cirurgião-dentista em ambientes clínicos e hospitalares.

$\S 10$ Dada a sua formação, o Técnico em Saúde Bucal é credenciado a compor a equipe de saúde, desenvolver atividades auxiliares em Odontologia e colaborarem pesquisas.

No Brasil, os TSBsse destacam com ampla força por compor a Equipe de Saúde da Família, a qual foi criada com base na consolidação do Sistema Único de Saúde (SUS $)^{7}$. Essas equipes trabalham de acordo com os princípios da Atenção Primária à Saúde, como, por exemplo, a integralidade à saúde ${ }^{19}$ compondo uma estratégia de organização dos serviços de saúde no país ${ }^{16}$.

As políticas do Ministério da Saúde e Ministério da Educação para o fortalecimento e ampliação dos processos de mudança na Educação Permanente em Saúde propõem, entre várias estratégias, a ampliação do pensamento crítico e dinamizador em saúde, através de cursos de Educação a Distância para a formação de ativadores de processos de mudança bem como na sua contribuição na capacitação e formação de recursos humanosdestinados ao SUS.

\section{OBJETIVO}

Este trabalho teve como objetivo apresentar a experiência da utilização do ensino à distância como importante ferramenta no processo de formação ecapacitação de profissionais Técnico em Saúde Bucal, utilizando as Tecnologias de Comunicação e Informação, em parceria da Faculdade de Odontologia com o Laboratório de Telessaúde/UERJ.

\section{RELATO DE EXPERIÊNCIA}

O projeto piloto de capacitação foi desenvolvido a partir da parceria instituída entre a Coordenação de Saúde Bucal do Município do Rio de Janeiro e a Faculdade de Odontologia da UERJatravés do PróSaúde, no qual previa capacitações diferenciadas para profissionais, de acordo com a demanda da rede pública de serviços de saúde. Um total de 10 profissionais Auxiliares em Saúde Bucal (ASB) da rede públicarecebeu o curso de capacitação em Técnico em Saúde Bucal (TSB) dentro de um modelo que previu a participação de cirurgiões dentistas (CD) da rede. Os CDs atuaram como preceptores das áreas programáticas destes profissionais bem como os coordenadores destas. Os preceptores (CDs da rede) receberam orientações e um protocolo de preceptoria para atuar junto aos alunos. Além da capacitação, os preceptores da rede tiveram acesso à plataforma do curso para percorrer as atividades teóricas, práticas e fóruns de discussão. Os alunos do curso ainda realizaram módulos práticos mensais e provas teóricas no ambiente da unidade acadêmica.

A partir desta experiência, o curso de TSB já existente na FOUERJ passou a utilizar esta metodologia, agora com foco não em capacitação, mas sim formação. A plataforma MOODLE do Laboratório de Telessaúde UERJ é utilizada para a estruturação do curso com:atividades teóricas, vídeos, aulas, bibliografia de apoio, exercícios de fixação, fóruns de discussãocientífica e cronograma. As atividades teóricas são mais diversificadas e disponibilizadas para o aluno que, a qualquer momento, pode revê-las na plataforma. $\mathrm{O}$ curso possui carga horária 
de $1200 \mathrm{~h}$, prevista na legislação em vigor CFO n ${ }^{\circ} 86 / 2009$.

\section{RESULTADOS E CONCLUSÃO Impacto na Formação e no serviço de saúde}

A abordagem do ensino por meio da educação à distância conta com a presença do professor (tutor) na elaboração de materiais institucionais bem como no planejamento de outras estratégias. Importante salientar, não só a aproximação do professor à realidade do aluno em suas atividades extramurais como também dos preceptores que auxiliam estes alunos na rede pública e que tem carência de um espaço para dividir conteúdos ${ }^{2}$.

Disponibilizar as tecnologias de informação e comunicação (TIC) aos alunos e colocá-los diante de informações, problemas e objetos de conhecimento nem sempre são suficientes para envolvê-los em um processo de aprendizagem colaborativa. Énecessário a criação de um ambiente que favoreça a aprendizagem significativa ao aluno, "desperte a disposição para aprender, disponibilize as informações pertinentes de maneira organizada e no momento apropriado, promova a interiorização de conceitos construídos. Baseados nesta afirmação observamos as redes sociais, amplamente utilizadas, como um incentivo a aderência dos alunos ao projeto de aprendizagem ${ }^{5}$.

Participar de um ambiente virtual significa atuar nesse ambiente, expressar pensamentos, tomar decisões, dialogar, trocar informações e experiências e produzir conhecimento. Cada pessoa busca as informações que lhe são mais pertinentes, internaliza-as, apropria-se delas e as transforma em uma nova representação, ao mesmo tempo que transforma-se e volta a agir no grupo transformado e transformando o gru- po ${ }^{9}$.

Nessa abordagem de EAD, ensinar é organizar situações de aprendizagem, planejar e propor atividades, identificar as representações do pensamento do aluno, atuar como mediador e orientador, fornecer informações relevantes, incentivar a busca de distintas fontes de informações, realizar experimentações, provocar a reflexão sobre processos e produtos, favorecer a formalização de conceitos, propiciar a interaprendizagem e a aprendizagem significativa do aluno.

Aprender é planejar; desenvolver ações; receber, selecionar e enviar informações; estabelecer conexões; refletir sobre o processo em desenvolvimento em conjunto com os pares; desenvolver a interaprendizagem, a competência de resolver problemas em grupo e a autonomia em relação à busca, ao fazer e compreender. As informações são selecionadas, organizadas e contextualizadas segundo as expectativas do grupo, permitindo estabelecer múltiplas e mútuas relações e recursões, atribuindo-lhes um novo sentido que ultrapassa a compreensão individual.

O fomento à oferta de disciplinas com uso de tecnologias de informação e comunicação para cursos presenciais, por meio do oferecimento de suporte técnico e pedagógico a docentes e discentes, estimulando a recriação de novas metodologias pedagógicas, onde os mesmos poderão utilizar as diversas ferramentas tecnológicas através de ambientes virtuais de aprendizagem.

Através do inquérito sobre satisfação e inclusão no mercado de trabalho, os 10 alunos da turma piloto e 20 alunos da segunda turma responderam que recomendariam a modalidade do curso (100\% e $85 \%$ respectivamente). Além disso, o índice de 
aprovação dos alunos no serviço público de saúde foi de $80 \%$.

Devido à possibilidade dos alunos participantes, juntamente com os preceptores das unidades de saúde, em estabelecerem contato direto através de ferramentas de comunicação (redes sociais), suas dificuldades e compartilhamento de realidades torna maior o poder de resolutividade dos problemas vivenciados no serviço de saúde pública.

Neste relato podemos concluir que o ensino à distância é uma potente ferramenta na formação e/ou capacitação, não só de graduação ou especialização, mas também técnica na área de Odontologia.

\section{REFERÊNCIAS}

1. Albright, M., Graf, D., \& Simonson, M. (2007). Administración y Evaluación de laTecnologíaInstruccional y laEducación a Distancia. Manual del Curso ITDE 8012. (pp.9- 17).Miami, Florida: NOVA.

2. Almeida, M. E. B. (2002). Tecnologia de informação e comunicação na escola: novos horizontes na produção escrita. PUC/SP. Mimeo.

(2001).. Formando professores para atuar em ambientes virtuais de aprendizagem. In: Almeida, F. J. (coord). Projeto Nave. Educação a distância. Formação de professores em ambientes virtuais e colaborativos de aprendizagem. São Paulo: s.n.

3. Brasil. Parecer $n^{0} 460$, de 6 de fevereiro de 1975, MEC/CFE. Dispõe sobre a habilitação em nível de $2^{\circ}$ grau de Técnico em Higiene Dental e Atendente de Consultório Dentário. Diário Oficial da República Federativa do Brasil, PoderExecutivo, Brasília, DF, 1975.

4. Brasil. Ministério da Saúde. Lei $\mathrm{N}^{\mathrm{o}}$
11.889, de 24 de dezembro de 2008 . Publicado no Diário Oficial da União de 26 de dezembro de 2008.

5. Camargos Júnior, A.P. Processos interativos na EaD: mediação pedagógica através das TICs. http://www.pedagogia.com.br/artigos/in teracaonaead/, 2012.

6. Conselho Nacional de Educação, MEC. Câmara de Educação Básica. (2002).Diretrizes Nacionais para a Educação a Distância no âmbito da Educação Básica. Relatores: Gouveia, S F. \&Bizzo, N. M. V. CNE/MEC.

7. Cornwall A; Shankland A. Engaging citizens: Lessons from building Brazil's national health system. Social Science \& Medicine, v.66, n.10,p.2173-84, 2008.

8. Costa, I. A escola, o professor e as novas tecnologias.

http://www.pedagogia.com.br/artigos/n ovastecnologias1/, 2012.

9. Dodge, B. Active Learning on the Web.http://edweb.sdsu.edu/people/bdod ge/Active/ActiveLearning.html, 2012.

10. Dorego, E. \& M. Polo (1998). Sistema de Actualización Docente delProfesorado (SADPRO- UCV). Agenda Académica. Vol. 5 \#1.

11. ForGRAD (2002). Fórum Nacional de Pró-Reitores de Graduação das Universidades Brasileiras. Educação a Distância (EAD) na graduação: as políticas e as práticas. Curitiba.

12. Pereira AC. Odontologia em saúde coletiva: planejando ações e promovendo saúde. Porto Alegre: Artmed, 2003.

13. Pérez, A. (2002). Elementos para elanálisis de lainteracción educativa enlosnuevos entornos de aprendizaje, pixel-bit revista de medios y educación. Recuperado el 09 de diciembre de 2009, de: 
http://www.sav.us.es/pixelbit/articulos/ n19/n19art/art1904.htm

14. Prado, M. E. B. B \& Valente, J. A. (2002) A Educação a Distância possibilitando a formação do professor com base no ciclo da prática pedagógica. In: Moraes, M. C. Educação a distância: fundamentos e práticas. OEA/MEC, Unicamp, NIED.

15. Prensky, M Open Letter to the Obama Administration (in Educational Technology Mar-Apr 2009).

16. PUCCA J. A política nacional de saúde como demanda social. Ciência \&Saúde Coletiva, v.11 n.1, p. 243-246, 2006.

17. Rendeiro MMP, Vieira C, Medina DLT. Infocultura: uma barreira de acesso à educação permanente?,

18.Rizekp, http://www.fututo.usp.br/pages/sala/sala .html, 2012.

19. Starfield B, Shi L. Policy relevant determinants of health: an international perspective. Health Policy, v.60, n.3, 201-18, 2002.

\author{
ABSTRACT \\ Useofdistance learningas a toolfortech- \\ nicaltraininginoral health
}

The aim of thisstudy was to analyzeand present, through a case study, the applicability of Distance Learningas a toolin thetraining ofprofessionaltechnical level(Technical Oral Health) areaof Dentistry. Theproposed constructionof thecourse modelwas based on theneed forlarge-scale training, to meet therapid and progressiveimplementationof the NationalOral Health Politic. There were twopilotclasses, to verifycompliance andapplicability of the modelat a distance,to formnew professionals, andto meetthose whoare alreadyinsertedin the labor market. After completionof the twoclasses, $100 \%$ of the studentscompleted the course. Ingroup1, $100 \%$ would recommendthe course andthe class $2,85 \%$ would.About achievingpublic tender, countingthe twogroups, $80 \%$ were approved. Therefore, webelieve thatdistance educationisa powerful toolto meet the demandsfortechnical trainingin dentistry, in the perspectiveof the SUS andthesectoral legislation.

Descriptors: distance education, SUS, Public Oral Health, Information Technology, Technical Oral Health. 